\title{
Pac-Euglena: A Living Cellular Pac-Man Meets Virtual Ghosts
}

\author{
Amy T. Lam ${ }^{* 1,2}$, Jonathan Griffin ${ }^{2}$, Matthew Austin Loeun², \\ Nate J. Cira ${ }^{2,3}$, Seung Ah Lee ${ }^{2,4}$, Ingmar H. Riedel-Kruse ${ }^{1,2^{* *}}$ \\ ${ }^{1}$ University of Arizona, Department of Molecular and Cellular Biology, Tucson, USA; ${ }^{2}$ Stanford \\ University, Department of Bioengineering, Stanford, USA ${ }^{3}$ Rowland Institute at Harvard, Cambridge, \\ USA; ${ }^{4}$ School of Electrical and Electronic Engineering, Yonsei University, Seoul, Republic of Korea \\ *aymikins@gmail.com; ** ingmar@email.arizona.edu
}

\begin{abstract}
The advancement of biotechnology enabled the development of "biotic video games", where human players manipulate real biological samples for fun and educational humanbiology interactions. However, new design principles are needed to both leverage and mitigate biological properties (e.g., variability and stochasticity), and create unique play experiences that transcend traditional video games. This paper describes the implementation of Pac-Euglena, a biotic Pac-Man analog, where players guide live microscopic Euglena cells with light stimuli through a physical microfluidic maze. Through use of multi-modal stimuli, a mixed biology-digital-human reality is achieved, enabling cell interactions with virtual ghosts and collectibles. Through an iterative design process, we illustrate challenges and strategies for designing games with living organisms. A user study ( $\mathrm{n}=18$, conducted at a university event) showed that Pac-Euglena was fun, stimulated curiosity, and taught users about Euglena. We conclude with five general guidelines for the design and development of biotic games and HBI interfaces.
\end{abstract}

\section{Author Keywords}

Biological user interfaces; biotic games; Euglena gracilis; augmented reality; mixed reality; human-biology interaction (HBI).

\section{CSS Concepts}

- Human-centered computing User interface design; Applied computing Life and medical sciences;

\section{INTRODUCTION}

Biotic games, defined as games which involve human players interacting with real biological materials or processes, are a frontier in biotechnology and gaming $[10 ; 19 ; 27 ; 28 ; 34 ; 40$; 48]. Employed in various contexts ranging from citizen science [33] to informal education $[10 ; 19 ; 40 ; 48]$, these games provide novel player experiences, particularly due in

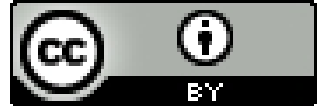

This work is licensed under a Creative Commons Attribution International 4.0 License.

CHI'20, April 25-30, 2020, Honolulu, HI, USA

(C) 2020 Copyright is held by the owner/author(s).

ACM ISBN 978-1-4503-6708-0/20/04

https://doi.org/10.1145/3313831.3376378 part to the semi-deterministic, noisy behaviors of living cells and materials. In educational applications, both real, physical setups as well as digital simulations have been found to have standout features when compared to each other, depending on educational goals and context $[6 ; 7 ; 13]$. While both types of systems deserve further development, here we focus on a system using real, living specimens. Living specimens can cause player emotions that are not achievable with purely digital games [22; 40], and increase user interest in biology compared to digital simulations and games [9]. Thus, biotic games have significant educational and entertainment potential $[4 ; 54]$.

The biotic game genre falls within the domain of HumanBiology Interaction (HBI - named in analogy to traditional human-computer interactions $\mathrm{HCI}$ ), an emerging HCI subfield involving biohybrid systems and mixed reality media $[11 ; 34 ; 36]$. Exploration of the design space for HBI and biotic games has only just begun. There are many open questions on how to leverage biological properties to achieve novel play experiences which are not attainable with traditional digital games, and how to design with and around the intrinsic limitations of biotechnology such as biological variability [19]. Also, many interesting and entertaining features and rules from traditional video games, such as restricted motion and temporary character disablement [43] have not been implemented in biotic games, in part due to hardware limitations: since current biotic games are primarily designed through virtual overlays [19], most biotic games do not allow for the virtual environment to affect the real-world local environment of the biological sample. Dealing with these design challenges and advancing the state-of-the-art for mixed reality media will stretch our ability to engineer and develop new hardware platforms for biological engineering $[11 ; 22 ; 33]$, sample stimulation $[31 ; 48]$, computer vision and object recognition $[2 ; 15 ; 49]$, and user interface design $[37 ; 48]$. 

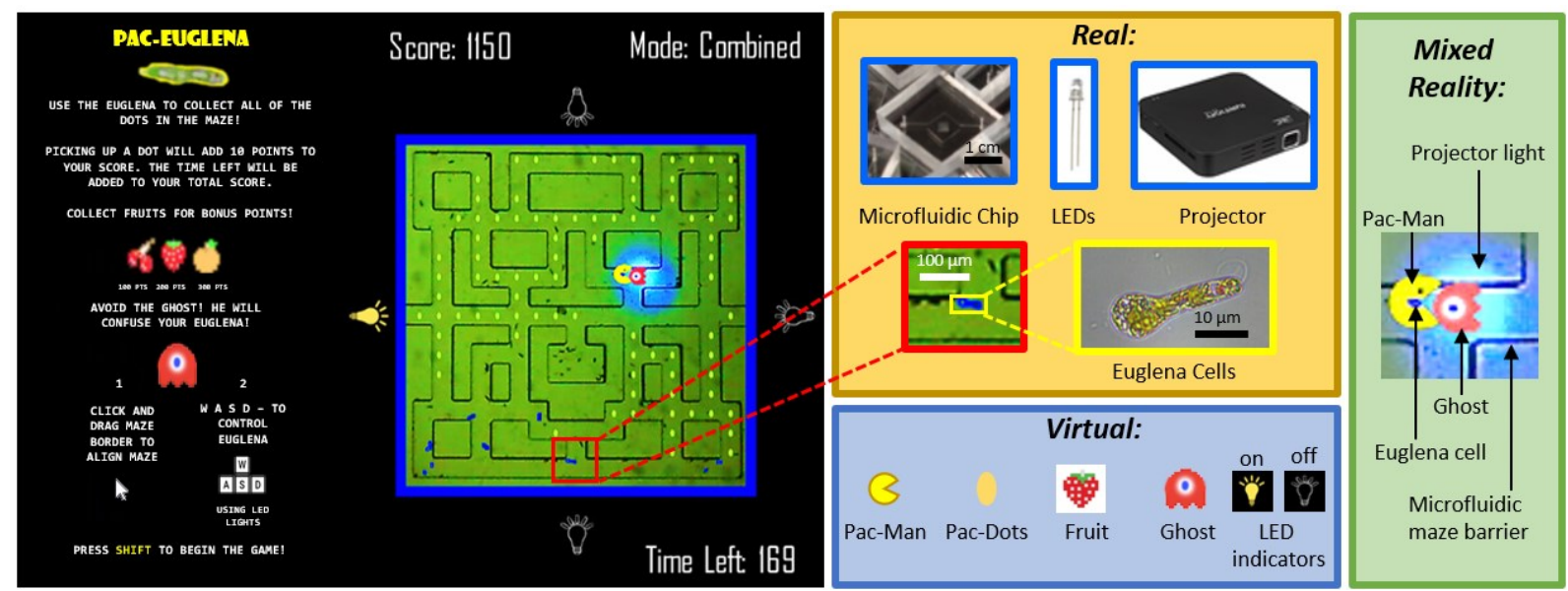

Figure 1. The Pac-Euglena game interface (left) with key hardware, biological, and software components (right). Players control live Euglena cells through WASD keys, which activate directional LEDs that steer the Euglena. Virtual (digital) objects are placed on top of the live microscope webcam feed. Computer vision is used to track cells in real time to interact with virtual objects. A projector perturbs cell movement during ghost encounters through localized light.

In this paper, we present the design rationale of a novel microfluidic environment for, and the iterative design process [43] and user tests of, a new biotic game "Pac-Euglena" (Fig. 1, Supplementary Video 1), inspired by the classic video game Pac-Man [38; 53]. Through this, we explore unique game design challenges and opportunities regarding the interaction design space of biotic games. Specifically, our goals were to (1) investigate the efficacy of complex microfluidic mazes in conjunction with local and global light stimuli and (2) implement two-way feedback between the biological sample and the virtual environment thereby creating a true mixed reality game for both the player and the living sample. We achieved both goals in Pac-Euglena, where human players steer live Euglena gracilis cells (unicellular phototactic microbes $[14 ; 57]$ ) via light stimuli through a microfluidic maze to collect and interact with overlaid virtual digital objects.

Our primary contributions are (1) the development of the first biotic game landscape realized through solid physical barriers inside a microfluidic chip; (2) the development of a two-way, mixed reality interaction between living cells (PacEuglena) and the digital overlay (the ghost); (3) the description of the iterative design process by which player feedback was integrated and addressed to develop three final game versions; (4) the development of various methods for working with biological variability, i.e., mitigating its detrimental effects on game robustness as well as enhancing player experience with natural biological behavior; and (5) the presentation of the user feedback from the final public display of Pac-Euglena, which combined with the iterative design process, resulted in general guidelines for biotic game design. These contributions will aid in the development of future biotic games, biotechnological user interfaces, and HBI in general.

\section{RELATED WORK}

Types of HBI have been expanded in recent years due to advances in biotechnology $[25 ; 30 ; 31 ; 33 ; 46]$. While human interactions with biological specimens on the macroscale have long been deliberately cultivated and designed (e.g., pet ownership, livestock and agriculture) $[3 ; 8 ; 18 ; 51]$, it was only relatively recently that microscopic biological specimens could be readily manipulated by non-specialists. With the development of more powerful and user-friendly computers, digital-biological hybrid systems were made, demonstrating the viability of having living organisms as both sensors and actuators for applications in materials science, design, art, and education $[23 ; 29 ; 35 ; 47 ; 50 ; 55$; 56]. More recently, direct interactions between human users and microbial specimens have been facilitated through biotic processing units (BPUs - biotechnological analogs of digital microprocessors like GPUs, with digital inputs and outputs) which enable both observation and stimulation of the biological samples, such as phototactic single-celled microbes $[10 ; 19 ; 25 ; 28 ; 31 ; 33 ; 34 ; 40 ; 48]$.

The integration of BPUs into computers allows them to take advantage of advances in live image processing and digital user interfaces, drawing HBI into the world of HCI. Digital overlays have both simplified and expanded HBI, enabling the incorporation of virtual objects in biotic setups $[10 ; 19$; 34; 40]. This enables the creation of mixed reality HBI, where real and virtual worlds merge and physical and digital objects coexist [41; 44]. However, biotic games have rarely featured digital objects which resulted in physical, real-world effects on the living specimen (Digital Action - Physical Effect transform (DPt)) [41]. More typically, the biological specimens are tracked using computer vision and affect the virtual overlay, but the virtual overlay does not affect specimen behavior; for example, in [27], cells could shoot a virtual soccer ball into a virtual goal, but the soccer ball and goal had no effect on the cells. A true mixed reality medium, where virtual objects also affect the environment around the specimen or the specimen's behavior, is more difficult to achieve. Nevertheless, such two-way interactions between the biological specimen and the digital virtual world [41] were realized in Metazoa Ludens [8], a mixed-reality tool for 
pet owners to remotely play with their pets through virtual avatars which meet on a virtual terrain; the virtual terrain reflected the motor-actuated changing terrain within the pet play area. Real, physical stimuli were also used to great effect in Cricket-Controlled Pac-Man [46], where a human player controlled the Pac-Man character on a virtual screen while living crickets, steered through surface vibrations, took the role of the ghosts. The crickets took the place of the computer-run character, and the virtual Pac-Man of the player was made real to the crickets via localized stimuli.

Creating mixed reality media for microscopic organisms presents unique challenges [46]. One such challenge is designing the interaction such that the biological specimen recognizes and is able to react to the user inputs and/or the virtual environment in a robust manner. It is difficult to tie together scales and perspectives for humans to meet much simpler and smaller organisms, which have very different sensory inputs or perceptions from humans [19]. Furthermore, creating microscale environments, localized stimuli, and user interfaces which bridge the large scale differential is technologically challenging.

Previous work on Euglena gracilis BPU development has solved many of these problems for Euglena-based systems [25; 31; 34]. References [10] and [25] established Euglena gracilis as a suitable organism for real-time experimentation and gameplay due to its relatively robust and quick responses to global LED light stimuli. Lee et al. bridged the size scale differential by enabling local light stimulation on the scale of a single cell by using a pico-projector controlled through a touchscreen [34]. This system was further extended through use of different user interfacing technologies [30]. The two stimuli modes were combined in [31], which used both local and global light stimuli in conjunction to steer ensembles of Euglena cells. Though simple microfluidic chips with very few structural barriers were employed in most of these systems, a deeper exploration of microfluidic chip design has not yet been done. In terms of mixed reality, the microbebased biotic games in References [27; 34; 40] involved virtual barriers and objects, but none of them allowed objects in the digital world to have an effect on the living specimens without direct intervention by the human player (e.g. the player physically places a trail of food on a petri dish).

\section{DESIGN CHOICES}

Our goal was to develop a playable and enjoyable biotic game and advance the state of the art of biotic systems for microorganisms by (1) developing the first complex microfluidic maze for a microbe-based BPU, allowing us to explore the limits and design principles for microfluidic chip design, and (2) achieving a true mixed reality medium with a two-way interactions between virtual objects and living specimens $[26 ; 27 ; 31 ; 34 ; 35 ; 40]$. We also wished to (3) explore the game design opportunities and challenges resulting from biological variability.

To these ends, we chose to implement a Pac-Man-like game. The Pac-Man maze and controls are well-suited for testing use of structured microfluidic chips with directional control of the cells. Additionally, the gameplay of the original PacMan game is relatively simple, which makes it easy to critically evaluate any changes in game design in our variation. Furthermore, a number of variations on Pac-Man have been developed in other HBI studies [27; 40; 46], which makes it easier to draw comparisons between our variant and other HBI variants. Finally, Pac-Man is well-known and recognizable by the average person and is thus more selfexplanatory. Our aim to implement a Pac-Man-like game provides a good first design goal from which we can explore how the limitations of our biotic system result in modifications to the original game play. This is especially interesting for designing games around biological variability rather than well-defined digital entities.

\section{CHOICE OF BIOWARE}

We wished to study biotic games (featuring real living biological organisms) rather than biological simulation games for several reasons. First, multiple studies have shown that user engagement with real systems can be better than with simulations, e.g., in classroom settings, students using real laboratory systems gain a more holistic view of the scientific process and technology involved $[6 ; 7 ; 13 ; 17]$. Specifically, in biotic systems, users report appreciation for their interactions with real living organisms, comparing it favorably to interactions with purely simulated entities, and they report greater interest, engagement, and emotional investment with the given tasks $[9 ; 22 ; 24 ; 26 ; 40]$. Secondly, by using a living organism, users are more likely to be motivated to explore and learn about biology and the enabling biotechnology, just as players of video and computer games grew into the next generation of computer scientists and programmers [20]. This is especially important as biotechnology becomes increasingly relevant to daily life and the economy [21]. Finally, by using a biological organism here, we have an opportunity to explore how such a system imposes unique design challenges. Just as the technological limitations of early video games drove game design, we expect that the limitations imposed by using a biotic system will likewise inform game design decisions [12]. These choices may only become apparent when implementing a game with real biological organisms.

\section{Logistical Considerations}

The choice of biological organism for biotic games must be approached carefully for practical reasons [19]. To limit the size and routine maintenance necessary for the setup, we choose to work with microbes. Using larger organisms would require an equivalently larger play area, which would make it more impractical to set up, especially in a home or casual setting. We also require that used organisms require minimal logistics for upkeep and maintenance. For example, mammalian cells would require regular exchange of media to prevent waste buildup and ensure that enough nutrients are there to keep the culture alive [42]. Furthermore, to allow users to interact with the organisms in real-time, the organisms must be able to respond to stimuli within seconds; otherwise, the interaction would be too slow to be 
appreciable by the user, and the game mechanics would suffer. For example, if slime mold was used in conjunction with nutrient pellets as the stimulus [26], players would need to wait minutes to hours between game actions, leading to a game with a very different dynamic.

\section{Ethical considerations}

Ethical considerations for biotic systems have already been studied in depth in [22], but we discuss them briefly here. Use of living organisms may prompt people to voice ethical concerns, often arising from anthropomorphization or fundamental misunderstandings about microbes. Objectively, single celled organisms are non-sentient and do not experience pain, and are of equivalent or lesser complexity to those regularly consumed by humans as food (e.g., yeast). For this work, we chose to require that the stimuli applied to our specimen should not kill them (e.g., natural predators), nor should it shorten the lifespan appreciably (e.g., strong UV light which may damage cells). The stimuli must be within the realm of what the organism would encounter in its natural habitat (e.g., sunlight, shade, physical obstacles). In comparison to many other practices with which humans regularly engage with other organisms (e.g. pet ownership, agriculture and raising livestock, fermentation, and genetic engineering), these criteria are stricter. First-hand engagement with microorganisms may enable people to educate themselves, confront and revise misconceptions, and develop a deeper understanding of the life sciences and biotechnologies.

By using photostimulation of the unicellular flagellate organism Euglena gracilis, we are able to address all of the above practical and ethical considerations. Euglena cells are $\sim 50 \mu \mathrm{m}$ long and $\sim 5 \mu \mathrm{m}$ wide and typically swim at $\sim 100$ $\mu \mathrm{m} / \mathrm{s}$. They exhibit negative phototaxis when exposed to bright light ( 200 lux $)$ though when suddenly exposed to very high light intensities (>3000 lux), they tend to spin in place [45]. Their responses to light occur within $\sim 1 \mathrm{~s}$, enabling direct interaction between the users and the cells. Previous work demonstrated the efficacy of Euglena in biotic setups $[19 ; 25 ; 28 ; 31 ; 34 ; 40 ; 49]$. Here we now explore methods combining stimuli on Euglena cells to create novel game mechanics in a mixed reality medium.

\section{HARDWARE IMPLEMENTATION}

The hardware setup is based on one presented in Reference [31] (Fig. 2). In brief, 5-50 Euglena cells are housed within a microfluidic chip. Directional light stimulation is provided by four LEDs placed on each side of the chip (Fig. 2). If an LED is turned on, cells will tend to swim away from the light [11]. Local light stimulation is provided by a projector, which illuminates the sample from below. The projected image is focused onto the sample plane such that spatially structured light stimulation can be achieved [17].

We developed the first biotic game where a microfluidic chip provides a game landscape (i.e., maze) that physically restricts in-game cell movement. We explored different chip designs, varying chamber height, maze layout, and channel width. We determined that the channel height and width needed to be large $(\sim 100 \mu \mathrm{m})$ compared to the cell dimensions to allow cells to move and turn freely. The maze area was $\sim 1.5 \mathrm{~mm} \times 1.5 \mathrm{~mm}$, and fit entirely within the field of view. Thus, if there were no barriers and the cell swim trajectory were straight, it would take about $15 \mathrm{~s}$ for a cell to swim across the maze (similar but slightly slower than classic Pac-Man, where the Pac-Man character takes $\sim 5 \mathrm{~s}$ to cross the screen). However, with the maze and the natural rotational noise of Euglena [45], a cell takes longer to move across the maze.

Initially, for recognizability, the maze was designed to exactly mimic the original level 1 Pac-Man. However, we later simplified the maze design (Figs. 1, 2) for two reasons: (1) cells do not respond well to narrow channels; and (2) we wished to match the physical distance between intersections with the average cells velocity to achieve a game dynamic similar to the original Pac-Man. To guarantee alignment of the digital overlay with the physical microfluidic chip, a calibration scheme was implemented on the software side.

We implemented temporary disablement of directional cell movement by direct local illumination of cells with a strong projector light; this induces cell spinning (Fig. 2) [45], which temporarily hinders the player's ability to control cell movement. This enabled the development of a computercontrolled antagonist, the ghost (Fig. 2). We determined that an illuminated area of $\sim 200 \mu \mathrm{m}$ diameter worked best for our purposes. The speed of the ghost was chosen to provide a reasonable game challenge. Thus, we were able to realize a mixed reality featuring two-way feedback between Euglena cells and virtually overlaid objects.

\section{GAME IMPLEMENTATION}

Here, we describe the final version of the Pac-Euglena Solo Mode (Fig. 1); our iterative design process and alternative play modes are described in the next section.

The player controls the four LEDs via the ' $W$ ','A', 'S', 'D'

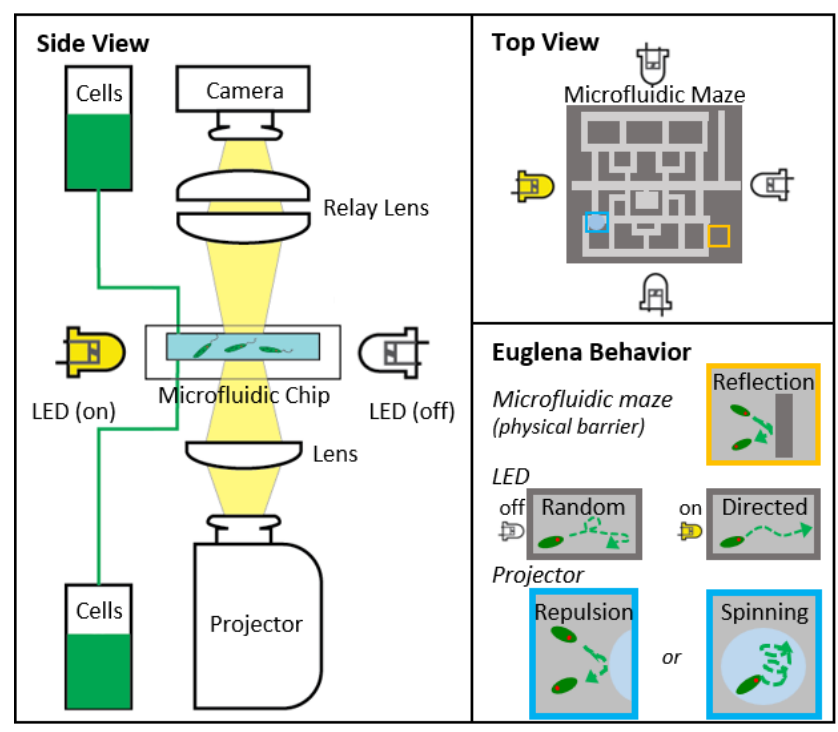

Figure 2. Hardware setup and Euglena behavior determine the physical and biological design space. 


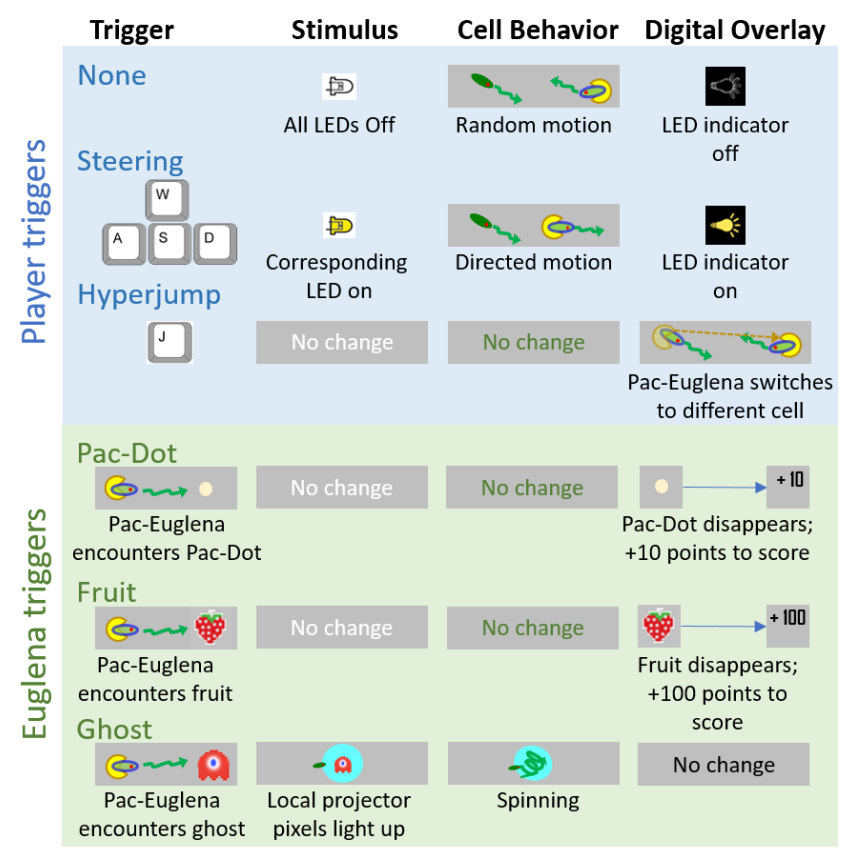

Figure 3. Triggers for all Pac-Euglena game events. Both human player and tracked cells trigger events, which affect the stimulus set, and in turn, affect cell behavior.

keys or a digital joystick to steer the Euglena through this maze (Figs. 1, 3). As the cells move, they "eat" the virtual "Pac-Dots" in the digital overlay (Fig. 3). The direction of the joystick or key presses corresponds to the expected motion of the Euglena rather than to the LED stimuli. For example, if the joystick is pulled to the left, the right LED turns on, and, barring any physical maze barriers, the Euglena are expected to move towards the left.

Single Euglena are tracked using computer vision allowing interactions with virtual objects, including point scoring (Fig. 3). The Pac-Man character is attached to one Euglena randomly at the start of the game. Whenever the tracked cell encounters a virtual Pac-Dot, it is removed on-screen and points are added to the player's score. On occasion, virtual "fruits" appear in the maze for a limited time and can be collected for extra points. Players can switch between the different cells by initiating a "hyperjump" by pressing ' $\mathrm{J}$ ', causing the Pac-Man avatar to switch to a different, randomly chosen cell (Fig. 3).

We also implemented a wandering virtual "ghost" that directly affects real Euglena behavior: If a tracked Euglena cell encounters the ghost, the area surrounding the ghost is illuminated by projecting strong blue light from below for $5 \mathrm{~s}$, which causes all illuminated cells to spin in place, i.e., they temporarily do not follow the directional LED stimulus and thereby slow down the player's progress (Fig. 3).

The ghost is a novel and conceptually significant game feature as it demonstrates usage of mixed reality in biotic games, where virtual objects (ghost) is made to affect living objects (cells) through a physical stimulus (projected light).

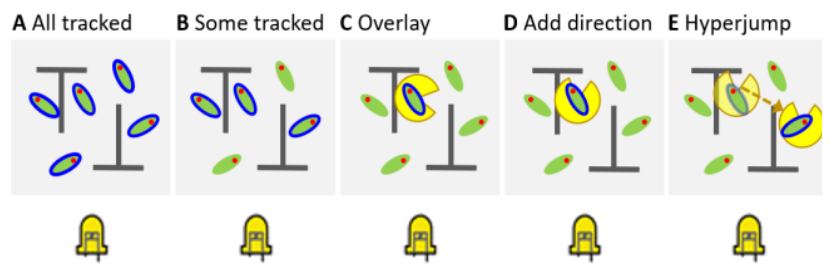

Figure 4. Successive stages for iterative game design.

The game ends after 3 minutes, or when all Pac-Dots are collected, which gives a score bonus.

\section{ITERATIVE GAME DESIGN}

\section{Motivation and Study Design}

Our goal for our iterative design process was, first, to develop Pac-Euglena into playable and enjoyable game, and second, to develop and evaluate solutions for dealing with any difficulties which arose from using living cells.

For each stage of the game design process, 2-4 volunteers played the game for 5-10 minutes and were asked to verbally provide feedback during and after gameplay. These volunteers were informally recruited from within the social groups of the game developers. All volunteers had worked in life science labs and had prior exposure to biotic games (i.e., had played or even helped develop biotic games before). Some volunteers provided feedback at multiple stages of the iterative design process. Volunteers often watched as other volunteers played, taking the part of spectator. Feedback was also requested from the spectators if available. This process ultimately led to the final versions of Pac-Euglena. Through describing our game design process, we showcase the strategies we used to work with the noise and stochasticity inherent to microbiological systems (Fig. 4).

\section{Results}

In the first game version, all cells within the microfluidic chip were tracked. Each cell tracked could score points by encountering a Pac-Dot (Fig. 3, 4A). However, players reported that the difficulty of the game greatly depended on the number of cells inside the maze since more cells enable faster collection of Pac-Dots. Typically, the number of cells ranged from 5 to 50 , and given the hardware setup, it was not feasible to always provide exactly one Euglena on the screen. Therefore, in the second version of the game, we decided that 10 randomly chosen cells would be tracked and could collect objects. In cases where there were fewer than 10 cells onscreen, the game would report an error and fail to start until a sufficient number of cells was detected. This made the game challenge more reproducible (Fig. 4B). Thus, by only using a subset of the available cells, we could increase consistency between successive rounds of game play.

The next issue was that spectators (i.e., bystanders), who play an important role in a gaming experiences [16], indicated that they found the game difficult to watch because they could not read the player's intention. When playing with multiple cells distributed over the maze, a player would typically focus on steering one Euglena for a while, and then focus on another 
cell in a different part of the maze where s/he considered the chance of collecting points as higher. However, bystanders would not know which Euglena the player was currently focusing on and what $\mathrm{s} / \mathrm{he}$ was trying to achieve. Therefore, in the third version of the game, we decided to overlay a virtual Pac-Man character over a single Euglena, and that only this Euglena would be able to score. As this hid the actual Euglena, we placed the outline of the tracked Euglena on top of the Pac-Man (Fig. 4C). We found that the digital overlay increased engagement of both the players and spectators.

However, while the player's and bystanders' attentions were now all successfully drawn to the same cell, the bystanders reported that it was difficult to tell in which direction the player intended to steer the cell. Thus, in iteration 4, we included the steering orientation in the Pac-Man overlay to signal the player intention: the Pac-Man position still corresponded to the cell position, but the mouth now pointed away from the light, i.e., in the direction of the intended cell motion (Fig. 4D). This illustrated to bystanders that the cell would sometimes move in a different direction than what the player intended. This led to amusement (Schadenfreude) among bystanders. Thus, we found that biological variability can contribute positively to the game experience.

Our test players indicated that playing a whole game with a single Euglena could become frustrating at times, especially in cases where the chosen Pac-Man cell was less responsive to the light stimulus. Therefore, in the fifth version, we implemented a 'hyper-jump', where the player could press the ' $J$ ' key which moved the virtual Pac-Man overlay to another randomly chosen Euglena on the screen (Fig. 4E). This not only solved this issue, but also led to new game strategies, e.g., players making the Pac-Man jump across the play field away from a region without many objects left to collect. Thus, we found that giving the player greater power over the digital character overlay can help mitigate biological variability as well as enhance the play experience.

These design iterations resulted in the Solo Mode described in the previous section. However, some players reported that this mode was frustrating because they were unable to score quickly enough to feel sufficiently rewarded. We ultimately allowed players to choose between the three modes: "Solo" (Fig. 4E), "Swarm" (Fig. 4B), or "Combined". In Swarm Mode, any of the 10 tracked cells can eat Pac-Dots and fruits or encounter ghosts. In the Combined Mode, again, 10 cells are tracked and can interact with Pac-Dots, fruits, and ghosts, but only one of the tracked cells has the Pac-Man character attached (Pac-Euglena). Eating Pac-Dots and fruits with PacEuglena allows players to score twice as many points. For Combined Mode, the player cannot easily prevent the nonPac-Euglena cells from collecting Pac-Dots, aside from steering the ensemble of cells or using hyperjump frequently. The hyperjump option is available in both Solo and Combined Modes.

\section{USER EVALUATION IN A PUBLIC SETTING}

\section{Motivation and Study Design}

A user study was done at a public university event to assess game playability and enjoyment with a more diverse audience and to determine which game features were successful. Participants (i.e., players) volunteered by joining a queue. Verbal and written consent was obtained to use the data gathered for each participant (IRB protocol 18334).

Pac-Euglena was played via a remote desktop using a virtual joystick. Individual players were randomly assigned a game mode. During gameplay, players were observed, and verbal exclamations as well as player expressions were transcribed by a single observer. After gameplay, players took a short online survey that included 2 multiple choice questions and 7 short answer responses (see "Survey Responses").

The survey questions were designed in accordance to the stated user study goals. We aimed to take no more than 10 minutes of the players' time for the gameplay and survey combined. Apart from the two multiple choice questions, which were designed to quantify user interest in the Euglena and enjoyment of the game, the questions were deliberately left open-ended to prevent bias in the user responses. Both the observations of the players and their survey responses were categorized into response types (as reported below) by a coder, and the analysis was confirmed by a second coder.

\section{Results}

A total of 18 participants played Pac-Euglena at the public event (6 players per game mode; self-identified as male or female). Except for the first two users, all users elected to play only one game due to queue buildup. All players had prior experience in life science labs; one user had previous exposure to biotic games.

The observational data as well as the responses to the survey questions for each game mode are reported in aggregate below, since there were no statistically significant trends in how players answered the quantitative, multiple choice questions, nor in the counts of coded response categories for the short-response questions, based on the mode of gameplay. P1, P2,.., P18 denote players 1-18. One Swarm Mode player (P13) did not complete the survey.

\section{Observation During Gameplay}

In our real-time observations of the players, $9 / 18$ the players asked questions relating to the biology and technology behind the game as they were playing. Often these were questions pertaining to Euglena movement or response to the stimulus (e.g., "They go away from light, right?" (P9) and "The Euglena can't teleport to the other side!" (P11).) P1 and P8 asked if the game was actually happening elsewhere, since they did not see the hardware setup at the event. P9 asked how long the setup had been in use while P10 asked how long the cells could survive within the setup and both seemed impressed by the answer (a few months for both questions). P15 asked if there was anything else one could study with this game or system. These questions demonstrate 


\begin{tabular}{l|ccc} 
MODE & N & $\begin{array}{c}\text { MEDIAN } \\
\text { SCORE }\end{array}$ & $\begin{array}{c}\text { MEAN } \pm \\
\text { STDEV }\end{array}$ \\
\hline $\begin{array}{l}\text { SOLO } \\
\text { (MAX 2500) }\end{array}$ & 6 & 690 & $647 \pm 233$ \\
$\begin{array}{l}\text { SWARM } \\
\text { MAX 2500) }\end{array}$ & 6 & 1560 & $1612 \pm 263$ \\
$\begin{array}{l}\text { COMBINED } \\
\text { (MAX 5000) }\end{array}$ & 6 & 1690 & $1732 \pm 248$ \\
\hline
\end{tabular}

Table 1. Player score statistics for each mode.

that this game may pique user interest in biology and biotechnology.

Players often showed emotional investment with how the game was proceeding, including panicking when cells encounter the ghost (4/18) (e.g., "No! Not the ghost!" (P2) and "Go right, go right, avoid the ghost!" (P17)), or verbally urging the cells to move in a specific direction (4/18) (e.g., "Move up. Come on!" (P18)). Player body language often indicated focused engagement (10/18), such as leaning in towards the computer screen or furrowed brows, though as indicated by their survey answers, this may also have been due to the screen being small. Some players expressed frustration during gameplay $(9 / 18)$ due to cell responses (3/18) (e.g. "So slow!" (P12) and "The Euglena are just spinning around..." (P15)), cell tracking (4/18) (e.g. "Now I'm just a piece of debris and I don't move!" (P2) and "It's mildly annoying when I have a blue outline [on a cell],... and then it switches [to another cell]." (P14)), or confusion over gameplay (4/18) (e.g. "It's too confusing to control these lights!" (P3)). Players also experienced a sense of accomplishment when they felt they were doing well (e.g. P5 exclaimed "I think I got the highest score!", and P9 exclaimed "Yes!" after winning).

As expected, Solo mode players, who could score using only one Euglena, had the lowest scores while Combined Mode players, who had 10 scoring cells with one cell scoring double the points, had the highest scores (Table 1). Interestingly, the average scores of Swarm Mode and Combined Mode players were not significantly different, which indicates that including the special Pac-Euglena cell which scored double the points did not seem to make much difference in the difficulty. One Swarm Mode player and one Combined Mode player won (i.e. collected all Pac-Dots). Note that the maximum points are only achieved if all PacDots and all fruits are collected. The maximum score in Combined Mode is only achieved if the player uses only the Pac-Euglena cell to collect all Pac-Dots and fruits.

\section{Survey Responses}

Question 1: What did you find most engaging, and why?

This question aimed to give players the opportunity to name successful features of the game, as well as to help us gauge player enjoyment.

Thirteen out of seventeen players answered this question (4 players left this question blank). The most common answers involved either controlling the Euglena motion or observing the Euglena responses to the light stimuli (5/13). For example, P5 stated "Being able to control the Euglena was very exciting". Others (3/13) reported that simply watching the cells was the most engaging aspect. Four players found the game mechanics to be interesting. P17 (a solo mode player) specifically indicated that the hyperjump routine was interesting and useful given the limitations in cell tracking ("Good to periodically switch [Euglena], since debris can fool the tracking"). One user (P14 - also from a Solo Mode player) reported that $\mathrm{s} /$ he felt emotionally attached to the cell ("I felt a kind of connection to my [Euglena]").

From these answers, it seems that the biotic nature of the game was highly appreciated, and that the root of the entertainment value was the fact that the cells were real, living organisms. The fact that some players reported that they enjoyed simply watching the cells may imply that our specific choice of organism was successful in that the cells appeared active and interesting enough on their own. Though P14 indicated that an attachment was formed with the living organism, the player did not report any ethical concerns.

Question 2: What did you find most challenging, and why? Since designing Pac-Euglena to be robust and enjoyable while taking biological variability into account was a major part of our iterative process, we wished to see if players noticed the biological variability of the cell responses in the final version, and gauge to what degree the variability interfered with their enjoyment of the game. We also wished to determine which game features were less successful.

Thirteen out of seventeen players answered this question (4 players left this question blank). Almost all answers to this question indicated that players found the cells difficult to control. Some found it difficult because the controls were not intuitive to them (4/13), while others found the stochastic responses of the cells difficult to manage (7/13). One player ("Combined mode" player) reported difficulty in "[keeping] track of all the Euglena on the screen" (P4).

Thus, we see that the biological variability in cell responsiveness is noticed by the players and adds difficulty to the game. This should be considered when designing games with somewhat stochastic elements, lest they result in excessive player frustration. Players who found the controls unintuitive all suggested changing the UI later in the survey (see Question 7). One possibility is to have the controls correspond directly to the stimuli rather than the expected Euglena responses. This may be more intuitive, since the former is a guaranteed control while the latter is stochastic.

Question 3: What did you find most entertaining, and why? Like question 1, this question also aimed to distinguish successful features of the game and gauge player enjoyment. Since question 1 asked for the "most engaging" feature, implying that players should only name one thing, we wished to give the players an opportunity to name another feature.

Ten out of seventeen players answered this question ( 7 players left this question blank). Many players reported being most entertained by the fact that they were working with real, 


\begin{tabular}{l|ccc}
\multicolumn{1}{c}{ MODE } & N & $\begin{array}{c}\text { MEDIAN } \\
\text { SCORE }\end{array}$ & $\begin{array}{c}\text { MEAN } \pm \\
\text { STDEV }\end{array}$ \\
\hline SOLO & 6 & 4 & $4.0 \pm 1.1$ \\
SWARM & 5 & 4 & $4.0 \pm 0.0$ \\
COMBINED & 6 & 4 & $4.3 \pm 0.5$ \\
TOTAL & 17 & 4 & $4.1 \pm 0.7$
\end{tabular}

Table 2. Survey results for Question 4: "Playing these games made me want to learn more about Euglena" (Likert scale 15). All players of "swarm mode" rated the statement a 4 , resulting in a zero standard deviation.

living cells (P12, P18; also Q6:P1, P4-P7, P9, P11, P14). Four players indicated that simply watching the biology/cell responses in motion entertaining. Others $(2 / 10)$ seemed to find the ability to control the cells, specifically, entertaining. Interestingly, P14 answered "How the [Euglena] didn't really do what I wanted it to do", which suggests that the player enjoyed the non-uniformity and non-deterministic behavior of the cells.

These answers again indicate that interacting with actual living organisms adds to player enjoyment. At least one player seemed entertained rather than frustrated with the stochastic responses of the cells. It may be worthwhile in a follow-up study to see whether players react emotionally to delayed or stochastic responses differently when dealing with live cells vs. simulated/digital characters.

Question 4: Playing these games made me want to learn more about Euglena. (Likert scale 1-5; 1: Strongly disagree, 2: Somewhat disagree, 3: Neutral, 4: Somewhat agree, 5: Strongly agree)

Since one major reason for developing biotic games is their educational potential, we wished to gauge the educational potential of Pac-Euglena with this question.

All 17 survey responders answered this question. Sixteen players reported either somewhat agreeing (rating 4, 12/16) or strongly agreeing (rating $5,4 / 16$ ) to this statement (Table 2). All swarm mode players who completed the survey gave this statement a 4. Only one player who played "solo mode" rated this statement a 2 ("somewhat disagree", P14). There were no statistically significant differences between the ratings of the different modes (Table 2).

This indicates that this game, and possibly others, may be effective in sparking curiosity in players, especially given the generally positive emotional response players had to the Euglena, as seen in previous questions.

Question 5: I had fun playing these games (Likert scale 1-5) We wished to obtain a more quantitative measure for how enjoyable Pac-Euglena was to the players.

All 17 survey responders answered this question. All survey responses were either "somewhat agree" (rating 4, 9/17) or "strongly agree" (rating 5, 8/17). There were no statistically significant differences between the ratings of the different modes (Table 3).

\begin{tabular}{l|ccc}
\multicolumn{1}{c}{ MODE } & N & $\begin{array}{c}\text { MEDIAN } \\
\text { SCORE }\end{array}$ & $\begin{array}{c}\text { MEAN } \pm \\
\text { STDEV }\end{array}$ \\
\hline SOLO & 6 & 4 & $4.3 \pm 0.5$ \\
SWARM & 5 & 4 & $4.4 \pm 0.5$ \\
COMBINED & 6 & 4.5 & $4.5 \pm 0.5$ \\
TOTAL & 17 & 4 & $4.4 \pm 0.5$
\end{tabular}

Table 3. Survey results for Question 5: "I had fun playing these games." Likert rating with scale 1-5 (1: Strongly disagree, 2: Somewhat disagree, 3: Neutral, 4: Somewhat agree, 5: Strongly agree).

Though the ratings might be positively inflated due to pleasing bias, these responses indicate that the game was not negatively received.

Question 6: What did you enjoy about these games?

This was the last question designed to give players the opportunity to name successful features of the game, as well as to help us gauge player enjoyment. This question was left more open-ended to capture any other positive impressions or features that players wanted to share with us.

Fifteen out of seventeen players responded to this question ( 2 players left this question blank). Players reported appreciating that this game was based on Pac-Man, which they were familiar with (4/15). Many players appreciated that they were interacting with live cells (9/15). Interestingly, P14 mentioned that stochasticity ("unpredictability") of the cells made the game fun. P6 stated that "I could engage with a living thing that I communicated with but could not fully control", while P11 stated "working with live organisms". However, quite a few answers also mention direct control or manipulation of the cells (4/15). Despite this, there were no ethical concerns reported by the players. We also note that, all of the comments on players enjoying the similarity to PacMan were from Swarm Mode players.

These answers raise the possibility that systems which use living cells may generate a more collaborative spirit between the player and the cell-based character rather than a controlling one. However, more work would have to be done to explore this effect.

Question 7: What improvements do you think can be made? This question was designed to determine which game features were not successful and help us find design guidelines for future biotic games.

All 17 survey respondents answered this question. Some of the players who played in the Swarm or Combined Mode mentioned that having a larger screen or having a greater magnification would make it easier to see (3/17). Several players also mentioned desiring more optimized or effective cell tracking (4/17). Some players reported difficulty in determining which cells were being tracked/counted for scoring, and they suggested thicker outlines for the cells (2/17). Otherwise, players generally suggested UI improvements, for example, regarding the type of input device used or input/stimulus mapping (4/17). For example, P6 suggested that we should "Make it more clear which 
Euglena is mine in solo mode, e.g. increase the width of the border surrounding my individual Euglena. Ability to use arrow keys as controls/have a USB joystick." There was no clear mapping between comment and mode, i.e. all categories of suggested improvements occurred from all player modes.

Since there were no issues reported with the speed at which the game proceeded, future iterations of this game would likely rely on digital zoom rather than any adjustments to hardware to increase magnification. These answers also suggest that mapping the player input to the expected cell behavior was not necessarily intuitive for players.

Question 8: Did you learn something new about biology or technology while playing these games?

As with question 4, we wished to gauge the educational potential of Pac-Euglena.

All 17 survey respondents answered this question. Most players reported that they learned that Euglena were light responsive (15/17), and more specifically that Euglena are negatively phototactic (8/17). Some players provided even more details on cell behavior (3/17), e.g., P12: "I learned that even though Euglena typically react to light by moving away from it, they sometimes get confused or disoriented while doing so". One player noted learning that computer vision was difficult (P4), which is perhaps something that would not have come up if the game had been purely digital.

These answers indicate that the biotic platform can indeed teach players about biology. However, once the mechanism of cell stimulation was recognized, further information about Euglena was not consciously picked up by players (e.g., general patterns of cell motion like that they tend to have helical trajectories).

Question 9: Any other comments you would like to share? This question was included to give players a place to voice any other thoughts that came to mind, positive or negative.

Four players responded to this survey question. All answers generally demonstrated enthusiasm for the game, either by suggesting sharing this game more broadly (e.g. P6: "This was a cool idea - get in touch with the Exploratorium" and P7 "This is really cool and I think you should share on the internet..."), or simple praise (e.g. P17: "Great job!" and P18: "really cool idea"). P7 specifically mentioned sharing via Internet, which would only be possible with remote access, as it was set up for the user trials.

These answers indicate that Pac-Euglena made some players genuinely excited.

\section{User Evaluation Summary}

In general, the use of live cells was one of the most popular and successful features of the game, as it greatly increased player engagement and enjoyment. We saw from player reactions and survey responses that some players felt that they were controlling or manipulating cells while others demonstrated a more collaborative spirit. We postulate that such collaborative mind frames may be easier to achieve with living and stochastically responding organisms, which may seem to have some free will.

Despite the non-uniformity of the cell responses and the resulting player frustration, players reported enjoying the game. One player even reported that the stochasticity was a source of entertainment and was amused that the cells sometimes acted contrary to what the player wished them to do. Interestingly, though the hyperjump routine was initially developed to counter the variability in the biological response, it was noted as a successful feature in dealing with errors in the cell tracking algorithm.

Pac-Euglena also demonstrated educational potential. Players recognized that they were able to influence the cell motion during gameplay, and almost all players learned that the Euglena cells were phototactic. Some players asked further questions about the setup and the cells. This suggests that the Pac-Euglena game (and other future biotic games) are potentially well-suited for STEM education $[5 ; 39]$.

\section{DISCUSSION AND CONCLUSION}

Pac-Euglena is the first systematic implementation of design principles presented in [19], so this work revealed effects which arose from the implementation. As suggested by Gerber et al. [19], we developed a way to draw player focus to a single cell (the Pac-Euglena avatar overlay) and found that it also enabled spectators to better understand the player intent. We also allowed players to select which cell to use/focus on (the hyperjump routine) and found that it resulted in novel play strategies.

Compared to other HBI Pac-Man variants [27; 40; 46], PacEuglena was the first microbe-based game that featured a physical maze and a digital ghost which also affected the real world. Previous microbe-based Pac-Man variants [27; 40] featured purely digital game elements, such as the maze [27], Pac-Dots [27; 40], and ghost [40], due to technological limitations with the hardware setup. Cricket-Controlled PacMan [46] successfully implemented a two-way mixed reality. In contrast to Pac-Euglena, the player has an antagonistic interaction with the specimens, which play the ghost to the player's Pac-Man character. This may result in different player affect towards the organism. Furthermore, compared to Pac-Euglena, the hardware system is much larger due to the larger specimens used. This makes it more difficult to setup in informal, uncontrolled settings.

Pac-Euglena also advances the state-of-the-art for Euglenabased biotic games. As we developed this game, we tested different chip designs and found that the minimum width/height for microfluidic channels was $100 \mu \mathrm{m}$ for effective cell steering/movement. Employing the microfluidic maze enabled the exploration of other uses for projected stimuli, resulting in the ghost interaction (previously, projected stimuli were used only as leaky barriers to cell motion, affecting Euglena behavior in such a way as to preclude the ghost interaction $[31 ; 34])$. The microfluidic maze has a better effective spatial resolution $(5 \mu \mathrm{m}$ barrier width, compared to the $300 \mu \mathrm{m}$ barrier width for maximal 
cell responsiveness for projected barriers [31]), which allows more varied maze designs. Conceptually, the development of the feedback loop between computer and organism is quite significant in that it enables more options for game design. For example, adversarial interactions between human and computers can now be achieved since the computer can manipulate the reality surrounding the organism using a different set of stimuli.

The iterative development process revealed various game design options for increasing playability and consistency in the face of biological variation. Both the use of multiple cells for the game as well as the hyperjump routine were employed to make the game more robust to variability in the setup. Unexpectedly, the hyperjump routine also led to new play styles. The inclusion of a character overlay helped focus the player onto a single cell, and improved spectator experience. These changes addressed the unique design challenges and opportunities in creating robust and meaningful interactions with noisy systems and non-uniform, adapting organisms, which is useful for further biotechnology development in general.

The user study showed the Pac-Euglena was a playable and enjoyable game. Notably, players appreciated the biotic nature of the game, and reported that they found the biological variability both entertaining and frustrating. This suggests that developers should not strive to eliminate all biological variability, and instead incorporate variability as a standout feature that sets biotic games apart from traditional and video games. Nevertheless, giving the player the ability to select a specific cell (e.g., the hyperjump routine) helps mitigate excessive frustration with biological variability or other technological limitations.

The user study also helped us identify features which could be improved. One common suggestion was to increase magnification. We note that for biotic systems, the question of scale is important to consider carefully. One must take into account the size and speed of the organism to allow the players to respond meaningfully to the organism $[19 ; 30]$. Once the appropriate scale has been decided upon, there may be further technological limitations to consider when building the hardware. Players also commonly found the UI unintuitive and reported that they needed to figure out how to control the cells. We postulate that mapping directional control to the stimulus rather than the intended motion of the cells would be more intuitive, since controlling the stimulus is in-line with what is actually occurring. However, both approaches may be equally valid and interesting.

From this work, we draw the following generalizable design lessons: (i) Player frustration, whether stemming from unresponsive cells or technological limitations, can be mitigated by giving players more actionable choices, e.g., choosing what cell to follow [19]; (ii) In cases where the variability precludes a consistent enjoyable gaming experience (e.g., having too low or too high a cell density), using larger ensembles of variable agents is one possible method to average out the variability; (iii) Designing the UI such that player inputs correspond to the stimulus rather than the expected cell responses may be more intuitive both because it is more in line with what the player is actually attempting to do, and because cell responses are somewhat stochastic; (iv) Game scale needs to be carefully considered so that players can easily distinguish cells from other features in the FOV (e.g. debris, the digital overlay characters and icons, and microfluidic walls), but also find the cell motion and responses engaging [19; 30]; (v) Slight stochasticity in cell responses can entertain players and spectators, and can perhaps help players see the organisms as independent entities. These design principles are applicable to non-biotic games as well. For example, giving players more options for motion or possible solutions to problems mitigates frustration in digital games [12]. However, biotic games often involve greater constraints and limitations; for example, technological constraints cannot eliminate biological variability or ensure perfect organism tracking, and the hardware setup necessary to house, stimulate, and image the organism limits the scale for the game. This necessitates and motivates innovative game mechanics.

This work raises questions for future biotic game research. Do biotic games foster a broader range of questions about biotechnology than purely digital games? Do they inspire a more collaborative rather than controlling outlook on the characters or living organisms in general? Are stochastic or otherwise non-uniform responses necessary for engendering sympathy or empathy for these living organisms? This user study serves as a starting point for deeper inquiry into the efficacy and enjoyment of biotic games. A larger scale study within a broader population with proper controls would be necessary to answer these questions more conclusively.

We also note that though biotic games have the potential to encourage critical assessment of HBIs outside of the game [19; 22], Pac-Euglena was not designed to do so. Future games could be designed to focus on the living nature of the biological specimens, narratively or thematically, to determine how it affects player evaluation of HBI ethics.

Biotic games foster the development of user interest in and a deeper understanding of cell behaviors at a different level than simulations alone [32]. The microscopic nature and advancing biotechnology will make versatile mixed celldigital-human reality applications affordable [31], especially in comparison to macroscopic mixed reality games. These new gaming approaches can be used with many other biological systems and have great potential to address growing needs in formal and informal STEM education $[1 ; 5$; 39; 52]. The current limitations on these types of biological systems will drive innovative interaction and game design, and emphasize the opportunities for further technological development, just as digital games did for computers and electronics.

\section{ACKNOWLEDGMENTS}

We thank members of the Riedel-Kruse Lab and E. Lo. We acknowledge funding from NSF Cyberlearning (\#1324753) and the Korean Government (NRF-2019R1A4A1025958). 


\section{REFERENCES}

[1] American Association for the Advancement of Science, 1994. Benchmarks for Science Literacy. Oxford University Press, USA.

[2] A. Arbelle, J. Reyes, J.Y. Chen, G. Lahav, and T.R. Raviv (2018). A Probabilistic Approach to Joint Cell Tracking and Segmentation in High-Throughput Microscopy Videos. Medical Image Analysis, 47, 140 152.

[3] A. Beck and A. H. Katcher (1984). A New Look at PetFacilitated Therapy. Journal of the American Veterinary Medical Association, 184(4), 414-421.

[4] Z. Bilda, E. Edmonds, and L. Candv (2008). Designing for Creative Engagement. Design Studies, 29(6), 525540.

[5] R.W. Bybee (2013). The Next Generation Science Standards and the Life Sciences. Science and Children, 50(6), 7-14.

[6] S. Chen, 2010. The View of Scientific Inquiry Conveyed by Simulation-Based Virtual Laboratories. Comput. Educ., 55(3), 1123-1130.

[7] S. Chen, W.H. Chang, C.H. Lai, and C.Y. Tsai, 2014. A Comparison of Students' Approaches to Inquiry, Conceptual Learning, and Attitudes in SimulationBased and Microcomputer-Based Laboratories. Science Education, 98.

[8] A.D. Cheok, R.T.K.C. Tan, R.L. Peiris, O.N.N. Fernando, J.T.K. Soon, I.J.P. Wijesena, and J.Y.P. Sen (2011). Metazoa Ludens: Mixed-Reality Interaction and Play for Small Pets and Humans. IEEE Transactions on Systems, Man, and Cybernetics - Part A: Systems and Humans, 41(5), 876-891.

[9] K.P. Chien, C.Y. Tsai, H.L. Chen, W.H. Chang, and S.F. Chen (2015). Learning differences and eye fixation patterns in virtual and physical science laboratories. Computers \& Education, 82, 191-201.

[10] N.J. Cira, A.M. Chung, A.K. Denisin, S. Rensi, G.N. Sanchez, S.R. Quake, and I.H. Riedel-Kruse (2015). A Biotic Game Design Project for Integrated Life Science and Engineering Education. PLoS Biology, 13(3), e1002110.

[11] S. Cooper, F. Khatib, A. Treuille, J. Barbero, J. Lee, M. Beenen, A. Leaver-Fay, D. Baker, Z. Popović, and Foldit Players (2010). Predicting Protein Structures with a Multiplayer Online Game. Nature, 466(7307), 756-760.

[12] C. Crawford, 1984. The Art of Computer Game Design. McGraw-Hill, Inc.

[13] T. de Jong, M.C. Linn, and Z.C. Zacharia, 2013. Physical and Virtual Laboratories in Science and Engineering Education. Science, 340, 305-308.

[14] B. Diehn (1973). Phototaxis and Sensory Transduction in Euglena. Science, 181(4104), 1009-1015.
[15] S. Diez, F. Ruhnow, and D. Zwicker (2011). Tracking Single Particles and Elongated Filaments with Nanometer Precision. Biophysical Journal, 100(11), 2820-2828.

[16] K. Emmerich and M. Masuch (2018). Watch Me Play: Does Social Facilitation Apply to Digital Games? In Proceedings of the 2018 CHI Conference on Human Factors in Computing Systems, ACM, 1-12.

[17] E.E. Toth, B.L. Morrow, and L.R. Ludvico, 2009. Designing Blended Inquiry Learning in a Laboratory Context: A Study of Incorporating Hands-On and Virtual Laboratories. Innovative Higher Education, 33(5), 333-344.

[18] F. French, S. Baskin, B. Wallace, A.D. Cheok, A. Zamanzky, and E. Nannoni (2017). FarmJam 2017: Designing Enrichment for Farm Animals. In Proceedings of the Fourth International Conference on Animal-Computer Interaction, ACM, 1-6.

[19] L. Gerber, H. Kim, and I.H. Riedel-Kruse (2016). Interactive Biotechnology: Design Rules for Integrating Biological Matter into Digital Games. In Digital Games Research Association (DiGRA) and the Foundations of Digital Games, 1(13).

[20] L. Haddon and D. Skinner (1991). The enigma of the micro: lessons from the British home computer boom. Social Science Computer Review 9, 3 (Fall), 435-449.

[21] M. Hakovirta and L. Lucia (2019). Informal STEM Education Will Accelerate the Bioeconomy. Nature Biotechnology, 37, 103.

[22] H. Harvey, M. Havard, D. Magnus, M.K. Cho, and I.H. Riedel-Kruse (2014). Innocent Fun or "Microslavery"? Hastings Center Report, 44(6), 38-46.

[23] G. Hertz (2008). Cockroach Controlled Mobil Robot: Control and Communication in the Animal and the Machine. http://www.conceptlab.com/roachbot.

[24] Z. Hossain, E. Bumbacher, A. Brauneis, M. Diaz, A. Saltarelli, P. Blikstein, and I. H. Riedel-Kruse (2018). Design Guidelines and Empirical Case Study for Scaling Authentic Inquiry-based Science Learning via Open Online Courses and Interactive Biology Cloud Labs. International Journal of Artificial Intelligence in Education, 28(4), 478-507.

[25] Z. Hossain, E.W. Bumbacher, A.M. Chung, H. Kim, C. Litton, A.D. Walter, S.N. Pradhan, K. Jona, P. Blikstein, and I.H. Riedel-Kruse (2016). Interactive and Scalable Biology Cloud Experimentation for Scientific Inquiry and Education. Nature Biotechnology, 34(12), 1293-1298.

[26] Z. Hossain, X. Jin, E.W. Bumbacher, A.M. Chung, S. Koo, J.D. Shapiro, C.Y. Truong, S. Choi, N.D. Orloff, P. Blikstein, and I.H. Riedel-Kruse (2015). Interactive Cloud Experimentation for Biology: An Online Education Case Study. In Proceedings of the 33rd 
Annual ACM Conference on Human Factors in Computing Systems, ACM, 3681-3690.

[27] H. Kim, L.C. Gerber, D. Chiu, S.A. Lee, N.J. Cira, S.Y. Xia, and I.H. Riedel-Kruse (2016). LudusScope: Accessible Interactive Smartphone Microscopy for Life-Science Education. PLOS ONE, 11(10), e0162602.

[28] H. Kim, L.C. Gerber, and I.H. Riedel-Kruse (2016). Interactive Biotechnology: Building Your Own Biotic Game Setup to Play with Living Microorganisms. In Proceedings of the 2016 CHI Conference Extended Abstracts on Human Factors in Computing Systems, ACM, 1000-1002.

[29] S. Kuribayashi and A. Wakita (2006). PlantDisplay: Turning Houseplants into Ambient Display. In Proceedings of the 2006 ACM SIGCHI International Conference on Advances in Computer Entertainment Technology, ACM, 40.

[30] A.T. Lam, J. Ma, C. Barr, S.A. Lee, A.K. White, K. Yu, and I.H. Riedel-Kruse (2019). First-Hand, Immersive Full-Body Experiences with Living Cells Through Interactive Museum Exhibits. Nature Biotechnology, 37(10), 1238-1241.

[31] A.T. Lam, K.G. Samuel-Gama, J. Griffin, M. Loeun, L.C. Gerber, Z. Hossain, N.J. Cira, S.A. Lee, and I.H. Riedel-Kruse (2017). Device and Programming Abstractions for Spatiotemporal Control of Active Micro-Particle Swarms. Lab on a Chip (17)8, 14421451.

[32] M.H. Lamers and W. van Eck (2012). Why Simulate? Hybrid Biological-Digital Games. In Proceedings of the 2012 European Conference on Applications of Evolutionary Computation, ACM, 214-223.

[33] J. Lee, W. Kladwang, M. Lee, D. Cantu, M. Azizyan, H. Kim, A. Limpaecher, S. Yoon, A. Treuille, R. Das, and EteR.N.A.Players (2014). RNA Design Rules from a Massive Open Laboratory. Proceedings of the National Academy of Sciences of the United States of America, 111(6), 2122-2127.

[34] S.A. Lee, E. Bumbacher, A.M. Chung, N. Cira, B. Walker, J.Y. Park, B. Starr, P. Blikstein, and I.H. Riedel-Kruse (2015). Trap it!: A Playful HumanBiology Interaction for a Museum Installation. In Proceedings of the 33rd Annual ACM Conference on Human Factors in Computing Systems, ACM, 25932602.

[35] S.A. Lee, A.M. Chung, N. Cira, and I.H. Riedel-Kruse (2015). Tangible Interactive Microbiology for Informal Science Education. In Proceedings of the Ninth International Conference on Tangible, Embedded, and Embodied Interaction, ACM, 273-280.

[36] D. Liu, C. Dede, R. Huang, and J. Richards (Eds). 2017. Virtual, Augmented, and Mixed Realities in Education. Springer Singapore.
[37] H. Lowood (2009). Videogames in Computer Space: The Complex History of Pong. IEEE Annals of the History of Computing, 31(3), 5-19.

[38] S. Mayer, L. Lischke, J.E. Grønbæk, Z. Sarsenbayeva, J. Vogelsang, P. Woźniak, N. Henze, and G. Jacucci (2018). Pac-Many: Movement Behavior when Playing Collaborative and Competitive Games on Large Displays. In Proceedings of the 2018 CHI Conference on Human Factors in Computing Systems, ACM, 1-10.

[39] H. Quinn, H. Schweingruber, T. Keller (Eds.). 2012. A Framework for K-12 Science Education: Practices, Crosscutting Concepts, and Core Ideas. The National Academies Press, Washington, D.C.

[40] I.H. Riedel-Kruse, A.M. Chung, B. Dura, A.L. Hamilton, and B.C. Lee (2011). Design, Engineering and Utility of Biotic Games. Lab on a Chip, 11(1), 1422.

[41] Y. Rogers, M. Scaife, S. Gabrielli, H. Smith, and E. Harris (2002). A Conceptual Framework for Mixed Reality Environments: Designing Novel Learning Activities for Young Children. Presence: Teleoperators and Virtual Environments, 11(6), 677-686.

[42] L. Sandell and D. Sakai (2011). Mammalian Cell Culture. Current Protocols Essnetial Laboratory Techniques, 5(1), 4.3.1-4.3.32.

[43] J. Schell. 2008. The Art of Game Design: A Book of Lenses. Morgan Kaufmann Publishers Inc., San Francisco, CA, USA.

[44] A.D.S.E. Silva and D.M. Sutko (2009). Digital cityscapes: Merging digital and urban playspaces. Peter Lang Publishing Inc.

[45] A.C.H. Tsang, A.T. Lam, and I.H. Riedel-Kruse (2018). Polygonal Motion and Adaptable Phototaxis via Flagellar Beat Switching in the Microswimmer Euglena Gracilis. Nature Physics, 14(12), 1216-1222.

[46] W. van Eck and M.H. Lamers (2006). Animal Controlled Computer Games: Playing Pac-Man Against Real Crickets. In Lecture Notes in Computer Science. Springer, Berlin, Heidelberg, 31-36.

[47] W. van Eck and M.H. Lamers (2013). Hybrid Biological-Digital Systems in Artistic and Entertainment Computing. Leonardo, 46(2), 151-158.

[48] P. Washington, K.G. Samuel-Gama, S. Goyal, A. Ramaswami, and I.H. Riedel-Kruse (2018). Prototyping Biotic Games and Interactive Experiments with JavaScript. In Extended Abstracts of the 2018 CHI Conference on Human Factors in Computing Systems, ACM, 1-4.

[49] P. Washington, K.G. Samuel-Gama, S. Goyal, A. Ramaswami, and I.H. Riedel-Kruse (2019). Interactive Programming Paradigm for Real-Time Experimentation with Remote Living Matter. Proceedings of the 
National Academy of Sciences of the United States of America, 116(12), 5411-5419.

[50] J. Wessnitzer, A. Asthenidis, G. Petrou, and B. Webb (2011). A Cricket-Controlled Robot Orienting Towards a Sound Source. In Proceedings of the 12th Annual Conference on Towards Autonomous Robotic Systems, ACM, 1-12.

[51] C.C. Wilson (1991). The pet as an anxiolytic intervention. Journal of Nervous and Mental Disease, 179(8), 482-489.

[52] C.D. Wilson, F. Reichsman, K. Mutch-Jones, A. Gardner, L. Marchi, S. Kowalski, T. Lord, and C. Dorsey (2018). Teacher Implementation and the Impact of Game-Based Science Curriculum Materials. Journal of Science Education and Technology, 27(4), 285-305.

[53] M.J.P. Wolf (ed). 2008. The Video Game Explosion: A History from PONG to Playstation and Beyond. Greenwood Press.

[54] N. Yannier, K.R. Koedinger, and S.E. Hudson (2015). Learning from Mixed-Reality Games: Is Shaking a
Tablet as Effective as Physical Observation?. In Proceedings of the 33rd Annual ACM Conference on Human Factors in Computing Systems, ACM, 10451054.

[55] L. Yao, J. Ou, C.Y. Cheng, H. Steiner, W. Wang, G. Wang, and H. Ishii (2015). biologic: Natto Cells as Nanoactuators for Shape Changing Interfaces. In Proceedings of the 33rd Annual ACM Conference on Human Factors in Computing Systems, ACM, 1-10.

[56] L. Yao, J. Ou, G. Wang, C.Y. Cheng, W. Wang, H. Steiner, and H. Ishii (2015). bioPrint: A Liquid Deposition Printing System for Natural Actuators. $3 D$ Printing and Additive Manufacturing, 2(4), 168-179.

[57] S. Yoshikawa, T. Suzuki, M. Watanabe, and M. Iseki (2005). Kinetic Analysis of the Activation of Photoactivated Adenylyl Cyclase (PAC), a Blue-Light Receptor for Photomovements of Euglena.

Photochemical \& Photobiological Sciences, 4(9), 727731. 